\title{
CONTROL SYSTEM OF COMFORTABLE ENVIRONMENT FOR DRIVERS
}

\author{
Yoshinobu HASHIMOTO*, Satori ARIMITSU*, Tadashi SUGA*, Kiyoshi ITAO*, \\ Hiroshi HOSAKA ${ }^{*}$, Ken SASAKI ${ }^{*}$, Tsuyoshi NAKAGAWA ${ }^{* *}$, Taiji KAWACHI ${ }^{* *}$ \\ * Division of Environmental Studies, Graduate School of Frontier Sciences, Tokyo University \\ 7-3-1 Hongo, Bunkyo-ku, Tokyo 113-8656, JAPAN \\ e-mail: hasimoto@itao.pe.u-tokyo.ac.jp \\ ** Research Laboratories, DENSO CORPORATION \\ 500-1 Minamiyama, Komenoki-cho, Nisshin-shi, Aichi 470-0111, JAPAN \\ e-mail:tnakaga@rlab.denso.co.jp
}

\begin{abstract}
The system that can make the driver's environment comfortable is under development. In this study, we consider the driver's environment to be most comfortable when fatigue or drowsiness of a driver is minimum. Using a driving simulator, various biosignal data and the work performance of drivers are collected in order to find effective biosignals for judging their comfort level. Among those biosignals, eye movements might be especially useful. Therefore, we began by developing the control system of comfortable environment for drivers based on eye movements.
\end{abstract}

\section{INTRODUCTION}

In spite of improvement in the performance of cars, there are still many automobile accidents in recent years. Most of those accidents result from driver's mistakes. It is generally thought that such mistakes originate in disorder of the driver's condition, such as a feeling of fatigue and sleepiness. As far as economic losses are concerned, for example, the annual cost of sleep-related accidents is estimated to be between 43 billion and 56 billion dollars in the United States [1]. If driver's fatigue or drowsiness can be found at an early stage, it will be possible to inform him of a risk of accidents. It is also possible to work on the driver's environment to reduce his fatigue or drowsiness. Therefore, it is very useful to monitor the driver's condition and evaluate the degree of his fatigue or drowsiness in order to prevent accidents.

Firstly, we looked for biosignals from which fatigue or drowsiness can be estimated.

\section{DRIVING SIMULATOR}

An indoor driving simulator was built, using a $\mathrm{PC}$, a steering wheel for computer games, a video projector and a screen. A driving simulator is ideal for our research because it provides an environment that is safe. In this driving simulation environment, various biosignal data such as pulse, heart rate, eye movements, body temperature and perspiration of drivers are collected. The work performance and the appearance of drivers are also acquired. The work performance is estimated from the degree of the lane deviation and the speed fluctuation. The driver is assigned to keep on driving through the center of a lane on monotonous roads at $100 \mathrm{~km} / \mathrm{h}$. The driver's appearance is recorded with a video camera fixed in front of the driver.

Correlation analysis between the collected biosignals and the work performance has shown that eye movements are useful for the detection of drowsiness or fatigue. This is because a slowdown in eye movements judged from the power spectrum of eye movements was highly correlated with the work performance decrement, and the emergence of a slowdown was also consistent with the onset of drowsiness or fatigue judged from the video footage. Then, we calculated several indicators of a slowdown in eye movements.

The system that can constantly monitor driver's eye movements and control the driver's environment is under development. If any abnormalities are found, this control system automatically works on the driver's environment by using an air conditioner, alarm sound, music, light, etc, in order to reduce his fatigue or drowsiness and enhance the work performance. And the effectiveness of these means is under testing now.

\section{MEASUREMENT OF EYE MOVEMENTS}

Although there are several other methods for measurement of eye movements such as the VOG(video-oculography), the EOG(electro-oculography) method is chosen because it is popular and simple. Horizontal eye movements can be measured by placing the electrodes on the outer canthi of each eye with a common electrode in the center of the forehead (see Fig. 1). This is because the permanent potential difference between the cornea and the retina generates an electrical field in the tissues surrounding the eye.

Three disposable silver chloride electrodes and an 
instrumentation amplifier are always used for this measurement.

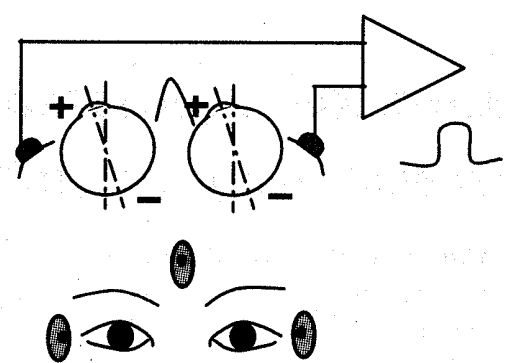

Fig. 1 EOG method of sensing eye movements

\section{EVALUATION OF EYE MOVEME}

Among the several indicators of a slowdown in eye movements, a ratio between the power in $0.1-30 \mathrm{~Hz}$ band and the power in $1-30 \mathrm{~Hz}$ band, which is denoted by $\mathrm{K}_{E T}$, has shown the highest correlation with the work performance decrement (see Eq. (1), Fig. 2 and Fig. 3).

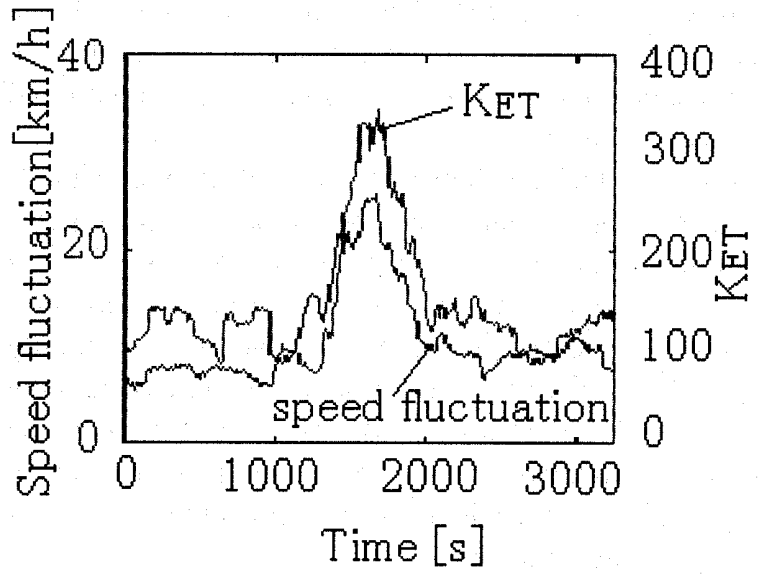

Fig. 2 Correlation between $\mathrm{K}_{E T}$ and the work performance (Speed fluctuation) decrement

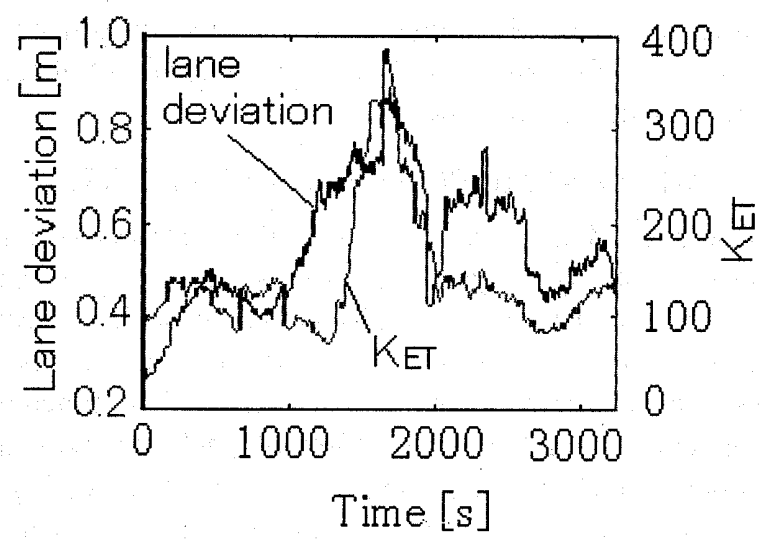

Fig. 3 Correlation between $\mathrm{K}_{E T}$ and the work performance (Lane deviation) decrement

$$
K_{E T}=\operatorname{Power}(0.1-30 \mathrm{~Hz}) / \operatorname{Power}(1-30 \mathrm{~Hz})
$$

By taking the common logarithm of the ratio of low frequency or high frequency, several other indicators were also calculated and their correlation with the work performance decrement was investigated.

\section{COMPACT EVALUATION DEVICE}

Using personal computers and analog devices, the control system of the driver's environment is taking shape now on the laboratory level. But our eventual goal is to build the control system in a real vehicle. Therefore, it is at least necessary for us to confirm the effectiveness of using a slowdown in eye movements outside the laboratory, even if confirmation while driving is somewhat difficult for safety reasons.

Then, we have built a small device that can be taken out of the laboratory and estimate the degree of a slowdown in eye movements by using a microcomputer module, some analog products, and a set of radio transceiver modules. The EOG data are pulse-width modulated and sent out from a digital transmitter. After reception, the data are demodulated trough a Low Pass Filter. This Low Pass Filter also serves as an Anti-Aliasing Filter. Then, the data are sampled at 100 $\mathrm{Hz}$ to be analyzed by the microcomputer module.

We plan to bring this device into a real vehicle to acquire the EOG data or evaluate a slowdown in eye movements while actually driving. Additionally, we are simplifying the algorithm for evaluation of a slowdown in eye movements, and are going to implement it on the LSI in order to make a wearable device for evaluation.

\section{CONCLUSION}

There is a high correlation between a slowdown in eye movements and the driver's work performance. By monitoring driver's eye movements it seems possible to keep the driver's environment comfortable and prevent accidents.

Although the EOG method with electrodes has been used in our study, hereafter we will also consider the possibilities of noncontact measurement of eye movements.

And, We are going to look for other effective biosignals in order to build a better system.

\section{REFERENCES}

[1] Leger, D., The Cost of Sleep-Related Accidents: A Report for the National Commission on Sleep Disorders Research, Sleep 1994, Vol. 17, pp. 84 - 93. 